\title{
COLOR OF DWARF GALAXIES IN THE COMA CLUSTER
}

S. OKAMURA ${ }^{1}$, Y. KOMIYAMA ${ }^{1}$, M. SEKIGUCHI ${ }^{2}$, N. KASHIKAWA ${ }^{3}$, K. SHIMASAKU ${ }^{1}$, M. YAGI ${ }^{1}$, M. DOI ${ }^{1}$, N. YASUDA ${ }^{1}$, W. KAWASAKI ${ }^{1}$, M. IYE ${ }^{3}$, D. CARTER ${ }^{4}$ AND B. MOBASHER ${ }^{5}$

${ }^{1}$ Department of Astronomy and Research Center for the Early

Universe, School of Science, University of Tokyo, Japan

${ }^{2}$ Institute for Cosmic Ray Research, University of Tokyo, Japan

${ }^{3}$ National Astronomical Observatory, Japan

${ }^{4}$ Royal Greenwich Observatory, UK, ${ }^{5}$ Imperial College, UK

We observed three 0.44 square degree fields centered on the Coma cluster center (Coma-1), about 1 degree SW of the Coma center (Coma-3), and on a control field in SA57 with the mosaic CCD camera at the prime focus of the 4.2-m William Herschel Telescope. We detected 5628, 5020, and 4323 galaxies down to $R=22 \mathrm{mag}$ in Coma-1, Coma-3, and SA57 fields, respectively. We measure the magnitude and color within the variable aperture $r_{90}$ in which about $90 \%$ of the total flux is included. The histograms of $(B-R)$ colors of galaxies are made for four magnitude bins of width $\Delta R=2 \mathrm{mag}$ covering $13<R<21 \mathrm{mag}$ for each of the three fields. The mean colors and the $1 \sigma$ scatters of the Coma galaxies are obtained by a histogram subtraction technique (Coma-1/3 minus SA57). We find a very shallow slope of the color-magnitude relation (CMR), $\Delta(B-R) / \Delta R=-0.0037$, which indicates nearly a constant $(B-R)$ color over 6 magnitude in $15<R<21$ mag $\left(-19.5<M_{R}<-13.5\right.$ at Coma cluster). Dwarf galaxies are dominant in this magnitude range, and we conclude that the mean color of dwarf galaxies in the Coma clusters is nearly constant at $(B-R) \sim 1.6-1.7$, which is similar to the color of the faint end of giant elliptical galaxies.

We investigate the effect of measuring apertures on the resulting CMR. We measure the color of Coma-1 galaxies $(R<18.7 \mathrm{mag})$ using fixed apertures of different sizes and derive the slope of the CMR. The slope of the CMR is found to become steeper for smaller apertures until the aperture size is comparable to the seeing size. The slope, $\Delta(B-R) / \Delta R$, changes from -0.035 at 5 arcsec aperture to -0.005 at 30 arcsec aperture. Accordingly, a quantitative statement of the CMR should be made with the quantitative definition of the measuring aperture. 